\title{
Perorally active nanomicellar formulation of quercetin in the treatment of lung cancer
}

\author{
This article was published in the following Dove Press journal: \\ International Journal of Nanomedicine \\ 7 February 2012 \\ Number of times this article has been viewed
}

\section{Bee-Jen Tan \\ Yuanjie Liu \\ Kai-Lun Chang \\ Bennie KW Lim \\ Gigi NC Chiu}

Department of Pharmacy, Faculty

of Science, National University

of Singapore, Singapore
Correspondence: Gigi NC Chiu Department of Pharmacy, Faculty of Science, National University of Singapore, 18 Science Drive 4, Singapore II 7543

Tel +6565165536

Fax +65 6779 I554

Email phacncg@nus.edu.sg
Background: Realizing the therapeutic benefits of quercetin is mostly hampered by its low water solubility and poor absorption. In light of the advantages of nanovehicles in the delivery of flavanoids, we aimed to deliver quercetin perorally with nanomicelles made from the diblock copolymer, polyethylene glycol (PEG)-derivatized phosphatidylethanolamine (PE).

Methods: Quercetin-loaded nanomicelles were prepared by using the film casting method, and were evaluated in terms of drug incorporation efficiency, micelle size, interaction with Caco- 2 cells, and anticancer activity in the A549 lung cancer cell line and murine xenograft model.

Results: The incorporation efficiency into the nanomicelles was $\geq 88.9 \%$ when the content of quercetin was up to $4 \% \mathrm{w} / \mathrm{w}$, with sizes of $15.4-18.5 \mathrm{~nm}$ and polydispersity indices of $<0.250$. Solubilization of quercetin by the nanomicelles increased its aqueous concentration by 110 -fold. The quercetin nanomicelles were stable when tested in simulated gastric ( $\mathrm{pH} 1.2)$ and intestinal ( $\mathrm{pH}$ 7.4) fluids, and were non-toxic to the Caco- 2 cells as reflected by reversible reduction in transepithelial electrical resistance and $\leq 25 \%$ lactose dehydrogenase release. The anticancer activity of quercetin could be significantly improved over the free drug through the nanomicellar formulation when tested using the A549 cancer cell line and murine xenograft model. The nanomicellar quercetin formulation was well tolerated by the tumor-bearing animals, with no significant weight loss observed at the end of the 10-week study period.

Conclusion: A stable PEG-PE nanomicellar formulation of quercetin was developed with enhanced peroral anticancer activity and no apparent toxicity to the intestinal epithelium.

Keywords: quercetin, polymeric micelles, PEG-PE, peroral drug delivery, lung cancer

\section{Introduction}

Cancer is a public health problem that affects many countries in the world. Globally, lung cancer is the leading cause of cancer death. In the treatment of advanced lung cancer, chemotherapy remains the mainstay; however, only modest increase in survival has been observed at the cost of significant toxicity to patients. ${ }^{1}$ It is imperative to search for new therapeutics with improved efficacy and safety profiles. In the anticancer drug discovery and development process, compounds with the highest anticancer activities often have bulky hydrophobic groups within their chemical structures, rendering them water-insoluble. ${ }^{2}$ Low water solubility not only gives rise to formulation difficulties but also serious therapeutic challenges. Administering the poorly soluble drug candidate intravenously might result in serious complications such as embolism and respiratory system failure due to drug precipitation, ${ }^{3}$ whereas poor absorption would result from extravascular dosing. ${ }^{4}$ 
The family of flavonoid compounds is one such example which has been shown to be promising for the wide applicability in the prevention and therapy of cancer, neurodegenerative, and cardiovascular diseases. Yet, the major hurdle in their clinical usage is poor water solubility, thus affecting their peroral biological activity. ${ }^{5}$ Among this family of agents, quercetin has been studied extensively for its anticancer activity, whereby it could inhibit a number of signal transduction targets important to cancer cell survival, proliferation, and metastasis. ${ }^{6,7}$ Quercetin progressed to early-stage clinical trials as an anticancer agent more than a decade ago; nevertheless, its administration required the use of solvents such as dimethylsulfoxide or ethanol. ${ }^{8}$ Chemical modifications have been attempted to improve quercetin solubility but could result in a loss of drug potency. ${ }^{9}$ Thus, alternative strategies based on the use of nanotechnologies have been undertaken to improve the water solubility and/or the bioavailability of quercetin so as to improve its biological activity. ${ }^{5}$ While most of the nanovehicles are designed with compositions sufficiently stable for intravenous administration, which is the most conventional route for cancer chemotherapy, orally active cancer chemotherapeutics have emerged as an attractive treatment modality because of patient preference, convenience in administration, and applicability in outpatient setting. ${ }^{10} \mathrm{~A}$ few studies have shown promising peroral antioxidant activity of quercetin when it was delivered by nanoscaled delivery systems, ${ }^{11,12}$ and recently, a lipid-based, submicron $(\sim 400 \mathrm{~nm})$ vesicular complex of quercetin has been developed with improved peroral anticancer activity as compared to a peroral quercetin suspension. ${ }^{13}$

In light of the advantages of peroral anticancer drug delivery and recent developments in the use of nanovehicles for the delivery of flavonoids, ${ }^{5}$ we have attempted to deliver quercetin perorally through the use of polyethylene glycol (PEG)-derivatized phosphatidylethanolamine (PE) as the diblock copolymer to form a self-assembled nanomicellar delivery system, and have compared the anticancer activity of this nanomicellar formulation with a peroral suspension of quercetin. The research on PEG-PE nanomicellar systems, pioneered by Torchilin and Alkan-Onyuksel, ${ }^{14-16}$ has contributed greatly to the intravenous delivery of water-insoluble agents. For instance, improved therapeutic efficacy of the intravenously administered PEG-lipid micelle formulation of paclitaxel has been demonstrated in tumor-bearing mice. ${ }^{15,16}$ The potential of PEG-lipid nanomicelles for the peroral administration of water-insoluble compounds has yet to be demonstrated in animal models.
Here, we have shown that the peroral anticancer activity of quercetin could be significantly increased when delivered in PEG-PE nanomicellar systems in the A549 human lung tumor xenograft model.

\section{Materials and methods \\ Materials}

1,2-distearoyl-sn-glycero-3-phosphoethanolamine-N[methoxy(polyethyleneglycol)-2000] (DSPE-PEG ${ }_{2000}$ ) and 1,2-distearoyl-sn-glycero-3-phosphoethanolamine-N[poly(ethyleneglycol)-2000-N'-carboxyfluorescein] (FITCDSPE-PEG ${ }_{2000}$ ) were purchased from Avanti-Polar-Lipids (Alabaster, AL). Cell culture media and supplements were from Gibco (Grand Island, NY). All other chemicals were purchased from Sigma-Aldrich Inc (St Louis, MO). A549, MDA-MB-231, and Caco-2 cell lines were purchased from the American Type Culture Collection (Manassas, VA).

\section{Preparation of nanomicellar quercetin}

The quercetin nanomicelles were prepared as follows: briefly, DSPE-PEG $_{2000}$ was dissolved in chloroform and quercetin in ethanol, the two organic solutions were mixed together according to the various weight proportions of drug and PEG-lipid. Subsequently, the organic phase was evaporated off under a stream of nitrogen gas to obtain a dried thin film. The film was further placed under vacuum for at least 3 hours to remove the residual solvent. Hank's balanced salt solution or phosphate-buffered saline (PBS) was used to hydrate the thin film to form the nanomicelles. For fluorescencelabeled nanomicelles, $1 \%$ of FITC-DSPE-PEG ${ }_{2000}$ was mixed with $99 \%$ of DSPE-PEG ${ }_{2000}$ (by mole) in chloroform, followed by evaporation and hydration as described above. Unincorporated quercetin was removed by centrifugation at $13,000 \mathrm{rpm}$ for 10 minutes followed by filtration through a $0.2-\mu \mathrm{m}$ filter. The resultant nanomicelle samples were frozen at $-86^{\circ} \mathrm{C}$ for 8 hours (ULT Freezer; Thermo Electron Corporation Forma, Waltham, MA,) and subsequently lyophilized at $-50^{\circ} \mathrm{C}$ to $-55^{\circ} \mathrm{C}$ for 48 hours at $5 \mathrm{~Pa}$ (Alpha $2-4$ LDplus Freeze Dryer; Christ, Osterode, Germany). No secondary drying was performed. Lyophilized samples were stored at $4^{\circ} \mathrm{C} \pm 2^{\circ} \mathrm{C}$ until further evaluation, and subsequently were reconstituted to original volume using milli-Q water as and when required. Drug concentration was determined by reading absorbance at $376 \mathrm{~nm}$ after mixing the micelle samples in ethanol, using a standard curve constructed with quercetin dissolved in ethanol. The size of the nanomicelles was determined by dynamic light scattering (Malvern Zetasizer 3000HS; Malvern, Worcestershire, UK). 


\section{Stability of nanomicellar quercetin in simulated physiological fluids}

The stability of quercetin nanomicelles were evaluated using two different aqueous media. The composition of the simulated gastric fluid (SGF) was as follows: $0.2 \% \mathrm{w} / \mathrm{v} \mathrm{NaCl}$ in $0.7 \% \mathrm{v} / \mathrm{v} \mathrm{HCl}, \mathrm{pH} 1.2$, and that of the simulated intestinal fluid (SIF) was as follows: $0.05 \mathrm{M}$ potassium dihydrogen phosphate/0.02 M sodium hydroxide, $\mathrm{pH}$ 7.0. The nanomicellar quercetin formulation was dialyzed in 1:5000 v/v in the respective simulated fluids for the specified duration. Subsequently, the formulation was analyzed for size and drug content as described above.

\section{Cell culture}

Cells were grown in humidified atmosphere of $5 \% \mathrm{CO}_{2}$ at $37^{\circ} \mathrm{C}$, with media changed every $3-4$ days. Caco- 2 cells were maintained in Eagle's minimal essential medium (EMEM) supplemented with $20 \%$ fetal bovine serum (FBS), $2 \mathrm{mM}$ L-glutamine, and 100 units $/ \mathrm{mL}$ penicillin and $100 \mu \mathrm{g} / \mathrm{mL}$ streptomycin. Stock cultures were grown in T-75 flasks. Upon $80 \%-90 \%$ confluency, cells were spilt using $2.5 \%$ trypsin containing $1.0 \mathrm{mM}$ EDTA. All cell culture media and reagents were obtained from Gibco. A549 and MDA-MB-231 cells were maintained in RPMI1640 medium supplemented with 10\% FBS, $2 \mathrm{mM}$ L-glutamine, and 100 units $/ \mathrm{mL}$ penicillin and $100 \mu \mathrm{g} / \mathrm{mL}$ streptomycin. Upon $80 \%-90 \%$ confluency, cells were spilt using $0.25 \%$ trypsin.

\section{Accumulation of nanomicelles in Caco-2 cells}

Caco-2 cells were seeded in 4-well Lab-tek chamber glass slides from Nalgene Nunc Inc (Naperville, IL) at a density of $7.2 \times 10^{4}$ cells/well and cultured for 21 days.

Subsequently, FITC-labeled nanomicelles were added to the cells and incubated for the specified duration at $37^{\circ} \mathrm{C}$. After incubation, cells were washed three times with ice-cold PBS before viewing under a Carl Zeiss fluorescence confocal microscope (Oberkochen, Germany) using $40 \times$ objective.

\section{MTT viability assay and lactose dehydrogenase (LDH) release assay}

The plating densities for A549 cells, MDA-MB-231 cells, and Caco- 2 cells were $5.0 \times 10^{3}$ cells/well, $3.0 \times 10^{3}$ cells/ well, and $1.33 \times 10^{4}$ cells/well, respectively, in the 96-well plates. A549 and MDA-MB-231 cells were incubated for 24 hours to allow attachment to plate before experimentation, while Caco-2 cells were incubated for 21 days to allow differentiation and tight junction formation. The medium was aspirated and replaced with serum-supplemented medium containing serial dilutions of quercetin in free (dissolved in ethanol) or nanomicellar form.

For MTT viability assay, the cells were treated at $37^{\circ} \mathrm{C}$ for the specified duration, and at the end, MTT was added to each well after medium removal and PBS rinsing to remove residual drug. Following a 4 -hour incubation at $37^{\circ} \mathrm{C}$, the well content was aspirated and $150 \mu \mathrm{L}$ DMSO was added to dissolve formazan crystals. Absorbance at $570 \mathrm{~nm}$ was measured with a Tecan SpectraFluorPlus reader (Tecan, Mannedorf, Switzerland). Cell viability was calculated as follows:

$$
\text { Viability }(\%)=\frac{\mathrm{Abs}_{\text {test }}-\mathrm{Abs}_{\text {background }}}{\mathrm{Abs}_{\text {vehicle }}-\mathrm{Abs}_{\text {background }}} \times 100 \%
$$

where $\mathrm{Abs}_{\text {test }}, \mathrm{Abs}_{\text {background }}, \mathrm{Abs}_{\text {vehicle }}$ represent the absorbance readings from the quercetin-treated wells, the medium-only wells, and the vehicle control wells, respectively.

For LDH release assay, treatment groups, and conditions were similar to those for MTT viability assay, except that the culture medium was collected at the end of treatment for LDH detection using Cytotox-One Homogenous Membrane Integrity Assay Kit (Promega Corporation, Fitchburg, WI) following the manufacturer's instructions. Fluorescence was measured using the Tecan SpectraFluorPlus plate reader with an excitation wavelength of $560 \mathrm{~nm}$ and emission wavelength of $590 \mathrm{~nm}$. Cytotoxicity was calculated as follow:

$$
\operatorname{Cytotoxicity~}(\%)=\frac{\mathrm{F}_{\text {test }}-\mathrm{F}_{\text {background }}}{\mathrm{F}_{\text {triton- } \mathrm{X}}-\mathrm{F}_{\text {background }}} \times 100 \%
$$

where $\mathrm{F}_{\text {test }}, \mathrm{F}_{\text {triton-X }}$ and $\mathrm{F}_{\text {background }}$ represent the fluorescence readings from the test condition (CNM or vehicle alone), triton-X $0.2 \%$, and background.

\section{Transepithelial electrical resistance (TEER) measurement in Caco-2 cells}

Cells were seeded at a density of $1.2 \times 10^{4}$ cells/well in collagen-coated filter membrane polycarbonate transwell inserts ( $12 \mathrm{~mm}, 1-\mu \mathrm{m}$ pore size, $0.3 \mathrm{~cm}^{2}$ growth area) obtained from BD Biocoat (Erembodegem, Belgium) and incubated at $37^{\circ} \mathrm{C}$ for 21 days. On day 21 , the baseline TEER values of Caco-2 cells were measured using Millicell ERS-2 Epithelial Volt-Ohm Meter, and inserts that record $>600 \Omega \mathrm{cm}^{2}$ were used for experiments. The chamber was washed twice with 
Hank's balanced salt solution before the addition of drug treatment. The cells were treated for 24 hours at $37^{\circ} \mathrm{C}$, and TEER values were recorded every 24 hours over the study duration of 96 hours.

\section{Animal efficacy study}

The animal study was conducted in the facilities of the British Columbia Cancer Research Center, with methodologies approved by the Institutional Animal Care Committee of the University of British Columbia, Vancouver, BC, Canada. The mice were cared for and used in accordance with the Canadian Council on Animal Care Guidelines. All mice used were between 20-22 g, and were housed in micro-isolator cages and given free access to food and water. Tumors were established in female Rag-2M mice by a single subcutaneous injection of $5 \times 10^{6} \mathrm{~A} 549$ cells (in $50 \mu \mathrm{L}$ ) in the lower back area. Tumor growth was monitored by caliper measurements along the length and width. Tumor volumes were calculated by the following formula: Tumor volume $=0.5 \times\left(\right.$ length $\times$ width $\left.^{2}\right)$. When the tumor size reached $50-100 \mathrm{~mm}^{3}$ (3 weeks post-tumor cell inoculation), mice were randomized into three study groups of six animals per group: (1) untreated control, (2) peroral quercetin suspension (vehicle $=25 \% \mathrm{v} / \mathrm{v}$ ethanol in water), and (3) peroral nanomicellar quercetin. The dose of quercetin was $30 \mathrm{mg} / \mathrm{kg}$, following a schedule of three times per week (Monday/Wednesday/Friday) for 3 weeks. Tumor size and body weight of the mice was monitored thrice weekly. Animals whose tumor size reached $1000 \mathrm{~mm}^{3}$, developed ulcerations, or displayed a weight loss of more than $5 \%$ were euthanized.

\section{Statistics}

Where appropriate, results are expressed as mean \pm SEM of at least three independent experiments. Statistical significance was assessed using one-way ANOVA followed by Fisher's least significant difference (LSD) test for multiple comparisons. A $P$ value of $<0.05$ was considered to be statistically significant. All statistical analyses were carried out using SPSS software (v 19.0; IBM, Armonk, NY).

\section{Results}

\section{Formulating quercetin into PEG-lipid nanomicelles}

The commonly used PEG-lipid conjugate, DSPE-PEG ${ }_{2000}$, was combined with quercetin in various weight proportions $\left(1 \%-5 \% \mathrm{w} / \mathrm{w}\right.$ of quercetin in DSPE-PEG $\left.{ }_{2000}\right)$. As shown in Table 1, the incorporation efficiencies of quercetin into the DSPE-PEG $_{2000}$ nanomicelles were found to be $88.9 \%$ and above when the content of quercetin was up to $4 \% \mathrm{w} / \mathrm{w}$. At $5 \% \mathrm{w} / \mathrm{w}$,
Table I Formulation attributes of nanomicellar quercetin

\begin{tabular}{lll}
\hline Quercetin (\% w/w) & Incorporation efficiency (\%) & Size (nm) \\
\hline 1 & $94.5 \pm 7.0$ & $17.9 \pm 2.0$ \\
2 & $93.1 \pm 3.6$ & $18.5 \pm 1.4$ \\
3 & $92.2 \pm 4.3$ & $17.6 \pm 0.9$ \\
4 & $88.9 \pm 5.6$ & $17.7 \pm 0.8$ \\
5 & $79.3 \pm 13.3$ & $15.4 \pm 1.4$ \\
\hline
\end{tabular}

the incorporation efficiency was reduced to $79.3 \%$. The size of the drug-loaded nanomicelles ranged from 15.4 to $18.5 \mathrm{~nm}$ (Table 1), and the polydispersity indices were $<0.250$ for all the nanomicellar formulations. The zeta potentials of the empty and drug-loaded nanomicelles were $-11.8 \pm 0.7 \mathrm{mV}$ and $-14.8 \pm 0.7 \mathrm{mV}$, respectively. The highest concentration of nanomicellar quercetin in Hepes-buffered saline that could be achieved without precipitation was $3 \mathrm{mg} / \mathrm{mL}$. This represents a 110-fold increase in the aqueous concentration of quercetin, calculated based on the aqueous solubility of free quercetin of $80 \mu \mathrm{M} .{ }^{17,18}$ Furthermore, nanomicellar quercetin could be processed into a powder by freeze-drying, without the need of a cryoprotectant or lyoprotectant. Reconstitution of the freeze-dried, nanomicellar quercetin with water did not result in a change in size or quercetin content (data not shown). Hence, subsequent evaluation of the nanomicellar formulation was based on the reconstituted nanomicellar quercetin.

\section{In vitro cytotoxicity of nanomicellar quercetin in the A549 lung cancer cell line}

The cytotoxicity of nanomicellar quercetin was evaluated in the A549 human lung cancer cell line. The highest concentration of quercetin evaluated was $100 \mu \mathrm{M}$, as drug precipitation was observed at higher drug concentrations.

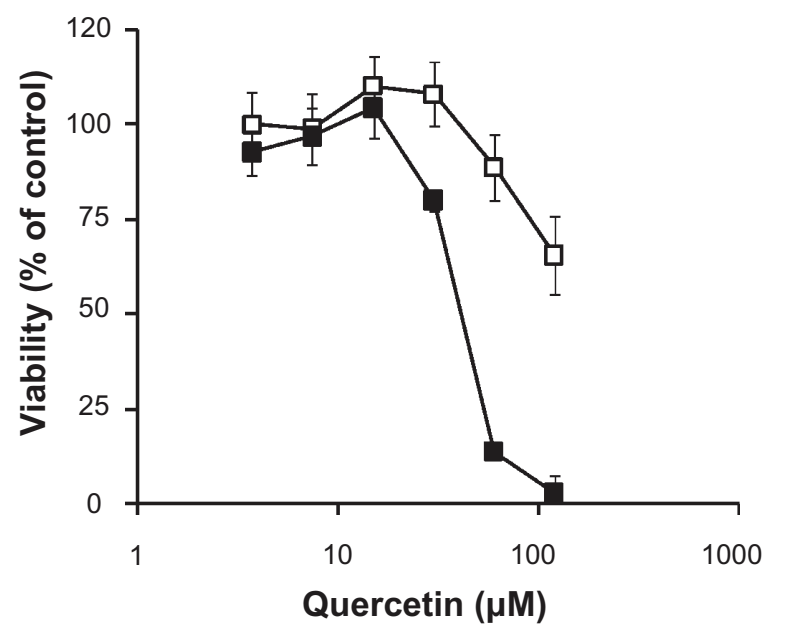

Figure I MTT viability of free $(\square)$ and nanomicellar $(\square)$ quercetin in A549 human lung cancer cell line upon 72 hours of exposure. 
As shown in Figure 1, the anticancer activity of quercetin could be significantly improved over the free drug through the nanomicellar formulation, as determined by the MTT viability assay. Of note, non-drug loaded DSPE-PEG ${ }_{2000}$ nanomicelles did not cause significant toxicity to the A549 lung cancer cells up to a concentration of $1 \mathrm{mM}$, with cell viability of $>85 \%$. To ensure that the cytotoxic effect was not cell line specific, the MDA-MB-231 human breast cancer cell line was treated with free and nanomicellar quercetin. As shown in Suppl Data Figure 1, a similar trend could be observed whereby the activity of quercetin could be significantly increased when formulated into the nanomicelles.

\section{Stability of nanomicellar quercetin in simulated physiological fluids}

Since the nanomicelles are to be administered perorally, stability in the fluids of the gastrointestinal tract has to be demonstrated. The stability of nanomicellar quercetin was evaluated over time in simulated gastric fluid (SGF, $0.2 \% \mathrm{w} / \mathrm{v} \mathrm{NaCl}$ in $0.7 \% \mathrm{v} / \mathrm{v} \mathrm{HCl}$, $\mathrm{pH} 1.2$ ) and simulated intestinal fluid (SIF, $0.05 \mathrm{M}$ potassium

\section{Stability in SGF (pH 1.2)}
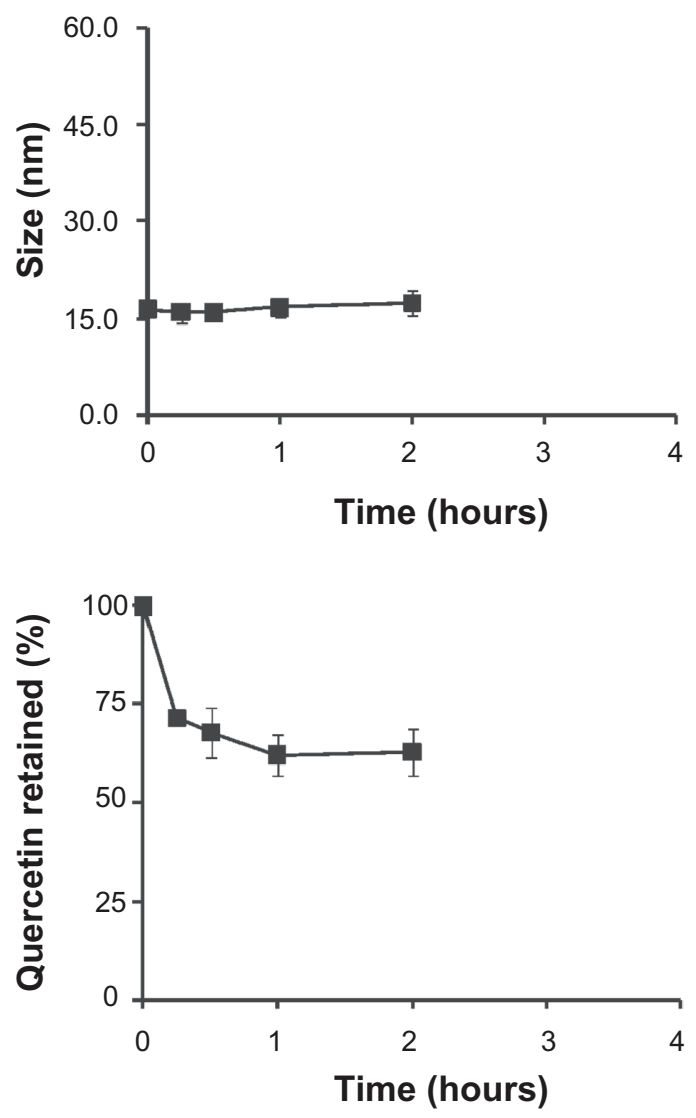

dihydrogen phosphate/0.02 M sodium hydroxide, $\mathrm{pH}$ 7.0). The quercetin nanomicelles were found to be stable, without significant change in size or drug precipitation (Figure 2). To investigate the amount of quercetin retained in the nanomicelles, the micelle samples were dialyzed against 1:5000 (v/v) SGF or $\mathrm{SIF}$ at $37^{\circ} \mathrm{C}$ using dialysis cassettes. Approximately $70 \%-75 \%$ of incorporated quercetin was retained in the nanomicelles in the respective simulated fluids (Figure 2).

\section{Interaction of nanomicellar quercetin with Caco-2 cell monolayer}

The well-established Caco-2 cell monolayer was employed as the in vitro model for gastrointestinal epithelium, and the cellular accumulation of the PEG-lipid nanomicelles was assessed by confocal microscopy. To facilitate visualization, the nanomicelles were spiked with 1 mol\% DSPE-PEG ${ }_{2000}{ }^{-}$ $\mathrm{N}$-carboxyfluorescein. The Caco- 2 cells were exposed to the fluorescence-labeled nanomicelles for up to 24 hours at $37^{\circ} \mathrm{C}$, and cellular accumulation of the nanomicelles increased over time (Figure 3A). Furthermore, the accumulation of
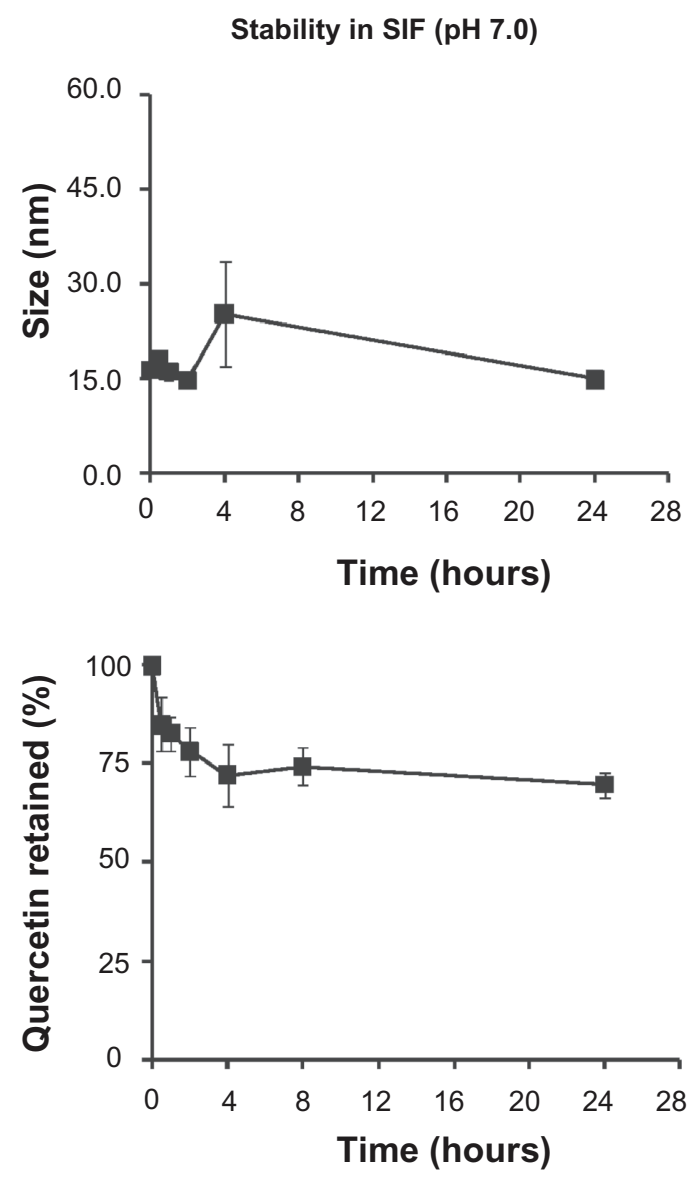

Figure 2 Stability of quercetin nanomicelles in simulated gastric fluid (SGF) and simulated intestinal fluid (SIF). 
the nanomicelles was independent of P-gp activity and endocytic processes, which was supported by results summarized in Suppl Data Figure 2. Cellular accumulation of the carboxyfluorescein-tagged nanomicelles occurred even in the presence of the P-gp inhibitor cyclosporine A or in the

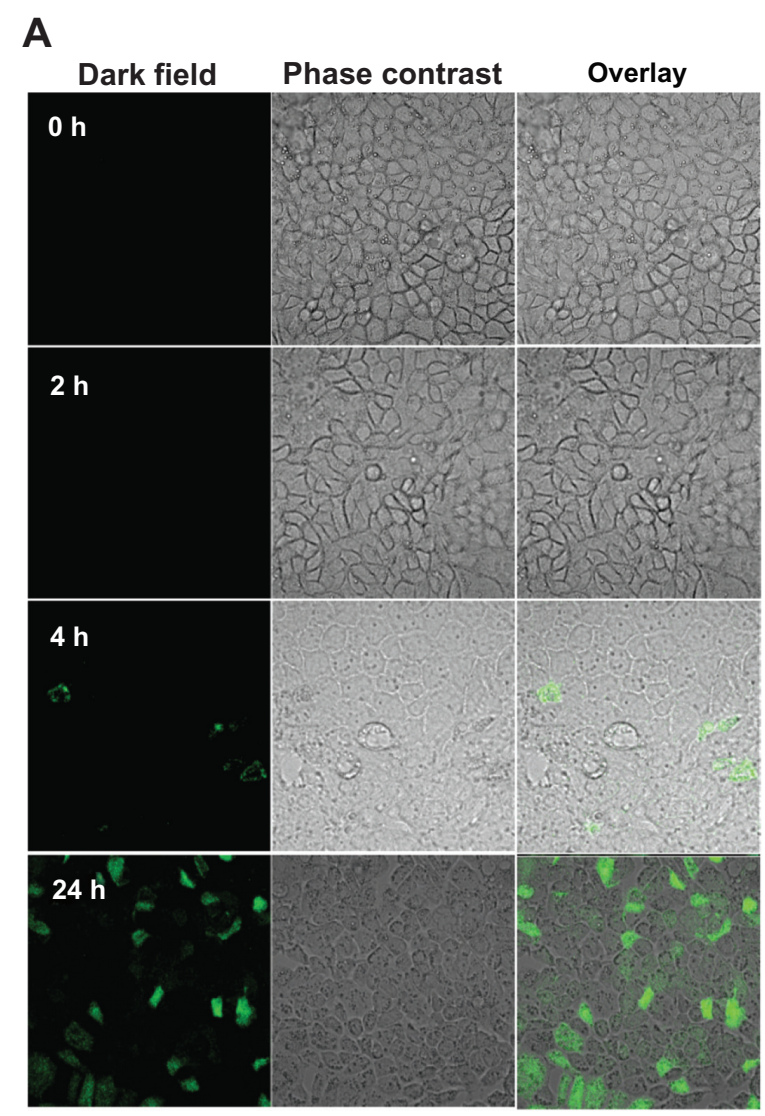

B

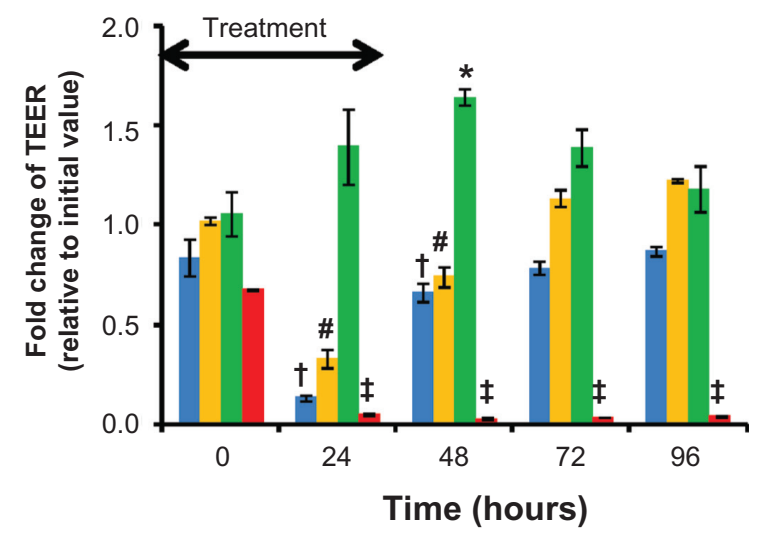

Figure 3 Interaction of quercetin nanomicelles with Caco-2 cells. (Panel A)Cellular accumulation of FITC-labeled nanomicelles over 24 hours of incubation at $37^{\circ} \mathrm{C}$ Representative images from fluoresence confocal microscopy are shown. (Panel B) Change in TEER upon treatment with quercetin in free (green) and nanomicellar (orange) forms, as well as empty nanomicelles (blue) and $1 \%$ triton-X (red), with the arrow indicating treatment duration of 24 hours.

Notes: ${ }^{+}, \#, *$, and ${ }^{\ddagger}$ represent $P<0.05$ compared to values at $\mathrm{t}=0$ hours. presence of endocytic inhibitors (hyperosmotic sucrose of $0.45 \mathrm{M}$ or Brefeldin $\mathrm{A}$ ) or a change of incubation temperature from $37^{\circ} \mathrm{C}$ to $4^{\circ} \mathrm{C}$. Collectively, these data suggest that the nanomicelles are likely to accumulate inside Caco-2 cells by passive diffusion.

One possible route to facilitate drug absorption through the intestinal epithelium is by disrupting the tight junctions and thus making paracellular transport of drug molecules possible, which could be reflected by a decrease in the TEER.${ }^{19}$ Nevertheless, it is also of equal importance that the disruption of the epithelial tight junctions is only transient, as irreversible disruption could represent potential toxicity to the intestinal epithelium. As such, differentiated Caco-2 cell monolayers grown in transwell inserts were exposed to free or nanomicellar quercetin for 24 hours, and TEER measurements were carried out over a time course of 96 hours. As shown in Figure 3B, Caco-2 cell monolayer treated with free quercetin had small yet statistically significant increase in TEER after 48 hours of exposure that gradually decreased back to control level ( $\mathrm{t}=0$ hours). This observation is in line with the protective effect of quercetin on the intestinal barrier function, which acts by promoting the assembly and expression of tight junction proteins. ${ }^{20}$ In contrast, significant reduction in TEER was observed in Caco-2 cell monolayer treated with nanomicellar quercetin during the first 48 hours. Of note, the reduction in TEER in the nanomicellar quercetin treatment group could be gradually increased back to control level ( $\mathrm{t}=0$ hours $)$, indicating the reversibility of the treatment.

To further investigate the potential toxicity of the nanomicellar quercetin formulation, release of LDH was determined in differentiated Caco-2 cell monolayer upon 24-hour treatment with free or nanomicellar quercetin (Figure 4).

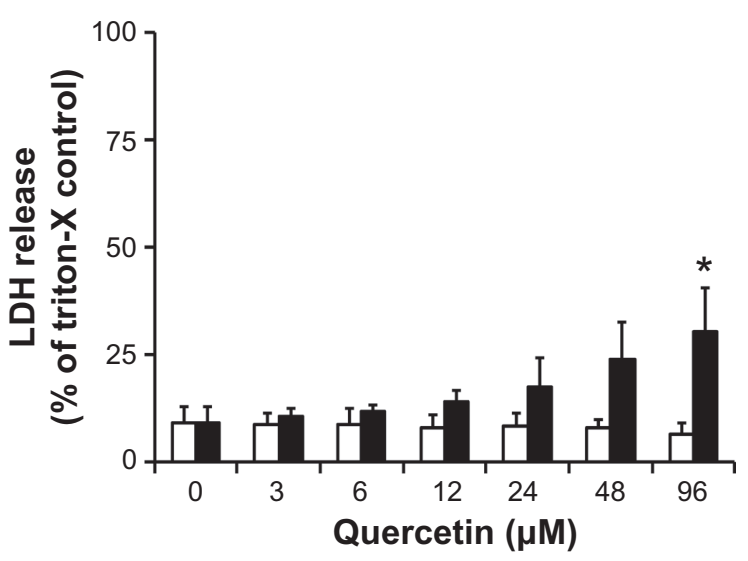

Figure 4 LDH cytotoxicity in Caco-2 cells when treated with free ( $\square$ ) and nanomicellar $(\square)$ quercetin for 24 hours. 
Treatment with free quercetin was non-toxic over the concentration range tested, with $\sim 10 \% \mathrm{LDH}$ released relative to triton-X control. In contrast, nanomicellar quercetin treatment yielded a small but significant increase in $\mathrm{LDH}$ release of $\sim 25 \%$ relative to triton-X control. Empty, nondrug loaded nanomicelles were evaluated at concentrations up to $1 \mathrm{mM}$ and were found to be relatively non-toxic to the Caco- 2 cell monolayer, with viability of $\sim 80 \%$ at $1 \mathrm{mM}$ (Suppl Data, Figure 3).

\section{In vivo efficacy of nanomicellar quercetin in human A549 lung tumor xenograft model}

Based on the in vitro evaluation of the nanomicellar quercetin formulation which provided promising results, the biological activity of the peroral formulation was further investigated in the A549 lung tumor xenograft model. Quercetin was administered to the tumor-bearing mice via oral gavage either in the nanomicellar form or in a $25 \% \mathrm{v} / \mathrm{v}$ ethanol-based suspension, with a dose of $30 \mathrm{mg} / \mathrm{kg}$ following a schedule of three times per week (Monday/Wednesday/Friday) for 3 weeks. The results demonstrated that the anti-tumor activity of quercetin was significantly increased when administered as the peroral nanomicelles compared to control and peroral quercetin suspension (Figure 5A). Specifically, tumor growth inhibition at 10 -week post-tumor inoculation was $83 \%$ for peroral quercetin suspension and $48 \%$ for peroral nanomicellar formulation. The two quercetin formulations were well tolerated by the animals, as reflected by minimal changes in the body weight of the mice (Figure 5B). No unusual observations were made upon necropsies of the animals from all three study groups.

\section{Discussion}

Quercetin, a flavanol that is found in certain vegetables, fruits, and tea, has been shown to have a range of biochemical and biological activities that could have the potential to translate to therapeutic benefits. In addition to its antioxidant and anti-inflammatory effects, quercetin as a tyrosine kinase inhibitor ${ }^{8}$ is able to modulate multiple signaling pathways that would have considerable implications in the therapeutic and prophylactic values for diseases such as cancer. However, realizing the biological benefits of quercetin in the clinical setting is mostly hampered by its low water solubility and poor absorption into the body. For instance, the baseline plasma concentration of quercetin from dietary intake is $50-80 \mathrm{nM},{ }^{21}$ and even with supplementation at $1 \mathrm{~g} /$ day for
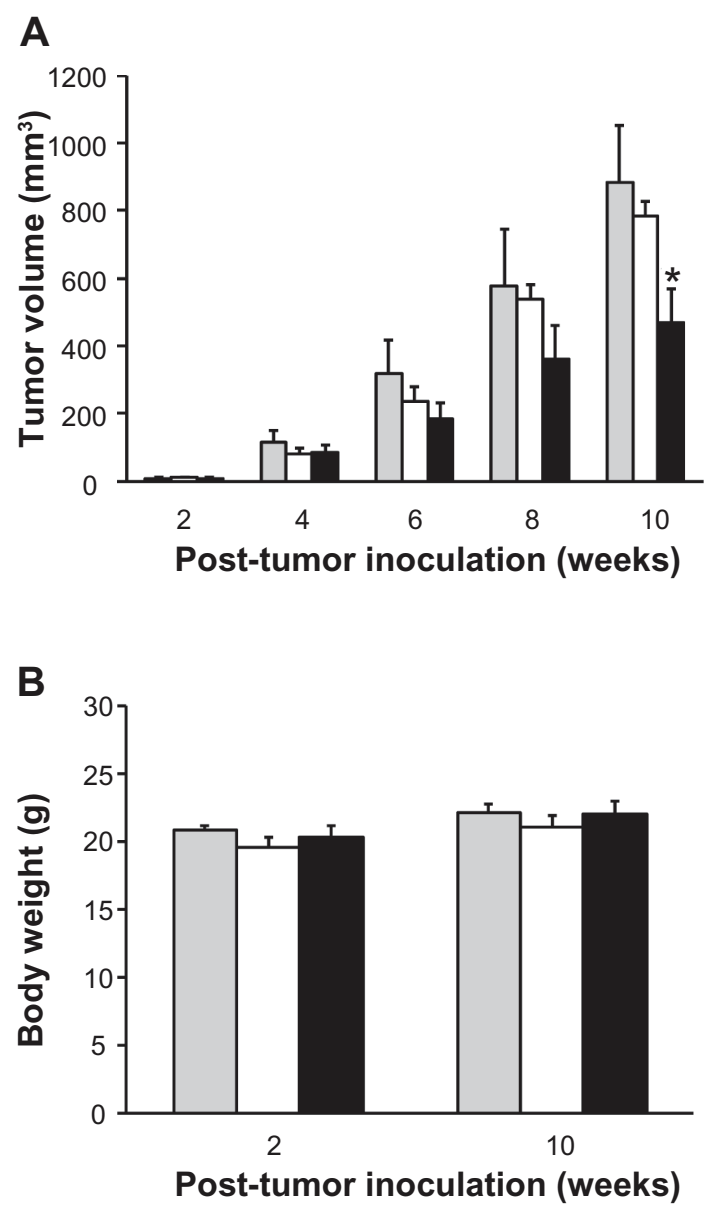

Figure 5 In vivo efficacy of orally given quercetin nanomicelles in subcutaneous A549 lung tumor xenograft murine model. Mice $(n=6)$ were treated with $30 \mathrm{mg} / \mathrm{kg}$ quercetin three times per week for 3 weeks by oral gavage, with treatment given in 3,4 , and 5 weeks post tumor inoculation. Study groups included untreated control $(\square)$, quercetin in ethanol-based suspension $(\square)$ or nanomicelles $(\square)$.

Notes: *represents $P<0.05$ compared to control and quercetin oral suspension.

28 days, the plasma concentration is $\sim 1.5 \mu \mathrm{M},{ }^{22}$ which is far below the concentration that is necessary to bring about the intended pharmacological effects of quercetin (such as the concentrations needed for anticancer effect presented in Figure 1). Thus, it is imperative to develop appropriate vehicles for quercetin, and it is the intention of our current study to develop a peroral nanomicellar system that could potentially improve its anticancer activity, which has not been extensively studied.

Since the quercetin-loaded DSPE-PEG ${ }_{2000}$ nanomicelles are intended for peroral application, the nanomicelles should be able to withstand the harsh environment of the digestive tract such as the drastic changes in $\mathrm{pH}$, and at the same time, be relatively non-toxic to the intestinal epithelium. As demonstrated in Figure 2, the quercetin nanomicelles were relatively stable at $\mathrm{pH} 1.2$ and $\mathrm{pH} 7$, with no significant changes in size and $\sim 30 \%$ release of quercetin. These findings are in good 
agreement with a previous study by Dabholkar et al which reported no significant change in the size of mixed micelles made of DSPE-PEG ${ }_{2000}$ and D- $\alpha$-tocopheryl polyethylene glycol 1000 succinate (TPGS) incubated in acidic (pH 1.2) and neutral ( $\mathrm{pH} 7.4$ ) conditions. ${ }^{23}$ In terms of toxicity to the intestinal epithelium, although the incorporation of quercetin into the nanomicelles has slightly increased the cytotoxicity, as reflected by the data in Figure 4, this level of cytotoxicity ( $\sim 25 \%$ as determined by $\mathrm{LDH}$ release) was comparable to those reported for TPGS and its analogs which are PEGderivatives well established for their ability to modulate peroral drug absorption. The Caco- 2 cytotoxicity of the TPGS analogs was reported to range from $20 \%-40 \%$, as determined by LDH release. ${ }^{24}$ Furthermore, quercetin-loaded nanomicelles did not induce irreversible reduction in the TEER of the Caco-2 epithelium (Figure 3B). This finding, together with those in Figure 4 and Suppl Data Figure 3, showed that the quercetin-loaded nanomicelles were relatively non-toxic, which is in line with another study reporting that empty or meso-tetraphenyl porphine loaded DSPE-PEG ${ }_{2000}$ micelles were minimally toxic to Caco- 2 cells. ${ }^{25}$

The therapeutic benefits of perorally administered quercetin is limited by poor absorption into the body that could be attributed to a number of reasons, including its chemical structure, low water solubility, and extensive metabolism and degradation in the gastrointestinal tract. ${ }^{26} \mathrm{Of}$ interest, quercetin has been shown to protect the intestinal epithelial barrier function by promoting the expression of a number of tight junction proteins and increase TEER of Caco-2 monolayer, ${ }^{27}$ implying that quercetin may limit its own absorption into the body by reducing paracellular permeability. Our current DSPE-PEG ${ }_{2000}$-based peroral nanomicellar formulation could increase the anticancer activity of quercetin by two possible mechanisms with supportive data: (1) increase in the aqueous concentration of quercetin (by 110 -fold to $3 \mathrm{mg} / \mathrm{mL}$ ) through solubilization in the nanomicelles, and (2) increase in permeability through modulation of the epithelial tight junctions as reflected by the transient decrease in TEER. At the molecular level, quercetin is able to increase the distribution of tight junction proteins, ZO-2, occludin, and claudin-1, to the actin cytoskeleton fraction, as well as increase the expression of claudin-4 in cytoskeleton fraction and in whole cell. ${ }^{27}$ From Figure 3B, it can be seen that empty nanomicelles could reduce TEER reversibly, and it is possible that the empty micelles could disrupt the assembly of the tight junction complex through the partition of DSPE-PEG ${ }_{2000}$ into the cell membrane bilayer.
Nevertheless, other possible mechanisms that could contribute to the increased activity of quercetin by the nanomicellar peroral formulation could not be ruled out. One such mechanism is the potential ability of the nanomicelles to protect quercetin from the enzymatic and degradative action in the intestinal tract. The metabolism of quercetin is complex, involving the formation of multiple metabolites by different pathways. ${ }^{28}$ Thus, future investigations on the full pharmacokinetic behavior of the nanomicellar formulation with metabolite profiling are necessary to gain insights if the nanomicelles could indeed protect quercetin from enzymatic metabolism in vivo. Currently, we are establishing mass spectroscopy-based analytical method to characterize the metabonomics of quercetin to address the question. Another possible mechanism is the increased mucoadhesion of the nanomicelles to the intestinal mucosa that could be provided by the PEG shell of the nanomicelles. ${ }^{29}$ Furthermore, it has been suggested that nanovehicles made of lipids could be taken up by lymphoid tissues in the intestinal tract which in turn could circumvent first pass metabolism. ${ }^{13}$

It is encouraging that the nanomicellar peroral formulation could enhance the anticancer activity of quercetin at a dose of $30 \mathrm{mg} / \mathrm{kg}$. It has been reported that a dose of $3 \mathrm{~g} / \mathrm{kg}$, which is 100 -fold higher than ours, did not cause any toxic or harmful effect to mice. ${ }^{30}$ Thus, future studies could titrate our current dose to higher levels, with the dosing frequency adjusted to daily dosing. The dose titration study could be further coupled to the examination of the correlation between tyrosine kinase inhibition and tumor growth inhibition, which could yield interesting pharmacodynamic data on this nanomicellar peroral formulation. Further evaluation could also include the potential of this nanomicellar peroral formulation as a chemotherapeutic and chemopreventive agent in animal models.

\section{Acknowledgments}

The authors would like to thank Dr Marcel Bally, Dr Dawn Waterhouse, and Ms Hong Yan (Department of Experimental Therapeutics, BC Cancer Research Center, Canada) for providing the facilities and technical support of the animal experiment. The research project is funded by Singapore Economic Development Board Biomedical Sciences Proofof-Concept Scheme (COY-15-POC/N197-9).

\section{Disclosure}

The authors declare no conflicting interests in connection with this article. 


\section{References}

1. Clinical Practice Guidelines: Non-small cell lung cancer. National Comprehensive Cancer Network 2008. Available from: http:// www.nccn.org/professionals/physician_gls/PDF/nscl.pdf. Accessed September 19, 2008.

2. Lipinski CA. Drug-like properties and the causes of poor solubility and poor permeability. J Pharmacol Toxicol Methods. 2000;44(1): 235-249.

3. Teicher BA, Andrews PA. Anticancer Drug Development Guide: Preclinical Screening, Clinical Trials and Approval. 2nd ed. Totowa, NJ: Humana Press; 2004.

4. Lipinski CA, Lombardo F, Dominy BW, Feeney PJ. Experimental and computational approaches to estimate solubility and permeability in drug discovery and development settings. Adv Drug Deliv Rev. 2001;46(1-3):3-26.

5. Leonarduzzi G, Testa G, Sottero B, Gamba P, Poli G. Design and development of nanovehicle-based delivery systems for preventive or therapeutic supplementation with flavonoids. Curr Med Chem. 2010;17(1):74-95.

6. Rahman I, Biswas SK, Kirkham PA. Regulation of inflammation and redox signaling by dietary polyphenols. Biochem Pharmacol. 2006;72(11):1439-1452.

7. Gupta SC, Kim JH, Prasad S, Aggarwal BB. Regulation of survival, proliferation, invasion, angiogenesis, and metastasis of tumor cells through modulation of inflammatory pathways by nutraceuticals. Cancer Metastasis Rev. 2010;29(3):405-434.

8. Ferry DR, Smith A, Malkhandi J, et al. Phase I clinical trial of the flavonoid quercetin: pharmacokinetics and evidence for in vivo tyrosine kinase inhibition. Clin Cancer Res. 1996;2(4):659-668.

9. Yuan ZP, Chen LJ, Fan LY, et al. Liposomal quercetin efficiently suppresses growth of solid tumors in murine models. Clin Cancer Res. 2006;12(10):3193-3199.

10. Bromberg L. Polymeric micelles in oral chemotherapy. J Control Release. 2008;128(2):99-112.

11. Priprem A, Watanatorn J, Sutthiparinyanont S, Phachonpai W, Muchimapura S. Anxiety and cognitive effects of quercetin liposomes in rats. Nanomedicine. 2008;4(1):70-78.

12. Ghosh A, Mandal AK, Sarkar S, Panda S, Das N. Nanoencapsulation of quercetin enhances its dietary efficacy in combating arsenic-induced oxidative damage in liver and brain of rats. Life Sci. 2009;84(3-4): 75-80.

13. Date AA, Nagarsenker MS, Patere S, et al. Lecithin-based novel cationic nanocarriers (Leciplex) II: improving therapeutic efficacy of quercetin on oral administration. Mol Pharm. 2011;8(3):716-726.

14. Torchilin VP. Micellar nanocarriers: pharmaceutical perspectives. Pharm Res. 2007;24(1):116.

15. Alkan-Onyuksel H, Ramakrishnan S, Chai HB, Pezzuto JM. A mixed micellar formulation suitable for the parenteral administration of taxol. Pharm Res. 1994;11(2):206-212.

16. Gao Z, Lukyanov AN, Chakilam AR, Torchilin VP. PEG-PE/phosphatidylcholine mixed immunomicelles specifically deliver encapsulated taxol to tumor cells of different origin and promote their efficient killing. J Drug Target. 2003;11(2):87-92.
17. Goniotaki M, Hatziantoniou S, Dimas K, Wagner M, Demetzos C. Encapsulation of naturally occurring flavonoids into liposomes: physicochemical properties and biological activity against human cancer cell lines. J Pharm Pharmacol. 2004;56(10):1217-1224.

18. van der Woude H, Gliszczynska-Swiglo A, Struijs K, Smeets A, Alink GM, Rietjens IM. Biphasic modulation of cell proliferation by quercetin at concentrations physiologically relevant in humans. Cancer Lett. 2003;200(1):41-47.

19. Schneeberger EE, Lynch RD. The tight junction: a multifunctional complex. Am J Physiol Cell Physiol. 2004;286(6):C1213-C1228.

20. Suzuki T, Hara H. Role of flavonoids in intestinal tight junction regulation. J Nutr Biochem. 2011;22(5):401-408.

21. Erlund I, Kosonen T, Alfthan G, et al. Pharmacokinetics of quercetin from quercetin aglycone and rutin in healthy volunteers. Eur J Clin Pharmacol. 2000;56(8):545-553.

22. Conquer JA, Maiani G, Azzini E, Raguzzini A, Holub BJ. Supplementation with quercetin markedly increases plasma quercetin concentration without effect on selected risk factors for heart disease in healthy subjects. J Nutr. 1998;128(3):593-597.

23. Dabholkar RD, Sawant RM, Mongayt DA, Devarajan PV, Torchilin VP. Polyethylene glycol-phosphatidylethanolamine conjugate (PEGPE)-based mixed micelles: some properties, loading with paclitaxel, and modulation of P-glycoprotein-mediated efflux. Int J Pharm. 2006;315(1-2):148-157.

24. Collnot EM, Baldes C, Wempe MF, et al. Influence of vitamin E TPGS poly(ethylene glycol) chain length on apical efflux transporters in Caco-2 cell monolayers. J Control Release. 2006;111(1-2):35-40.

25. Sezgin Z, Yuksel N, Baykara T. Investigation of pluronic and PEG-PE micelles as carriers of meso-tetraphenyl porphine for oral administration. Int J Pharm. 2007;332(1-2):161-167.

26. Passamonti S, Terdoslavich M, Franca R, et al. Bioavailability of flavonoids: a review of their membrane transport and the function of bilitranslocase in animal and plant organisms. Curr Drug Metab. 2009;10(4):369-394.

27. Suzuki T, Hara H. Quercetin enhances intestinal barrier function through the assembly of zonula [corrected] occludens-2, occludin, and claudin-1 and the expression of claudin-4 in Caco-2 cells. J Nutr. 2009;139(5):965-974.

28. Manach C, Williamson G, Morand C, Scalbert A, Remesy C. Bioavailability and bioefficacy of polyphenols in humans. I. Review of 97 bioavailability studies. Am J Clin Nutr. 2005;81(Suppl 1):230S-242S.

29. Sahlin JJ, Peppas NA. Enhanced hydrogel adhesion by polymer interdiffusion: use of linear poly(ethylene glycol) as an adhesion promoter. J Biomater Sci Polym Ed. 1997;8(6):421-436.

30. Ruiz MJ, Fernandez M, Pico Y, et al. Dietary administration of high doses of pterostilbene and quercetin to mice is not toxic. J Agric Food Chem. 2009;57(8):3180-3186. 


\section{Supplementary data}

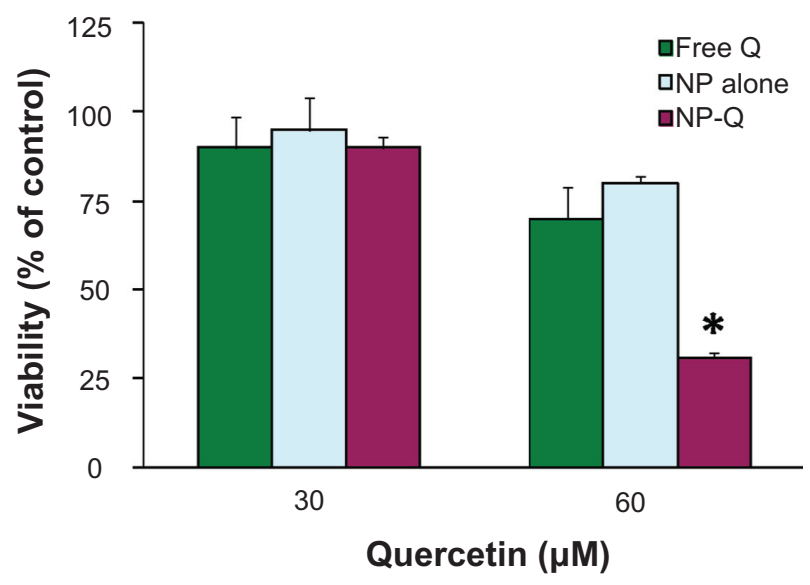

Figure SI MTT viability of free and nanomicellar quercetin in MDA-MB-23I human breast cancer cell line upon 72 hours of exposure. The concentrations of empty micelle were $96 \mu \mathrm{M}$ and $192 \mu \mathrm{M}$ for $30 \mu \mathrm{M}$ and $60 \mu \mathrm{M}$ nanomicellar quercetin, respectively.

Notes: Data represent mean \pm SEM from three independent experiments. $* P<0.05$ as compared to free quercetin group.
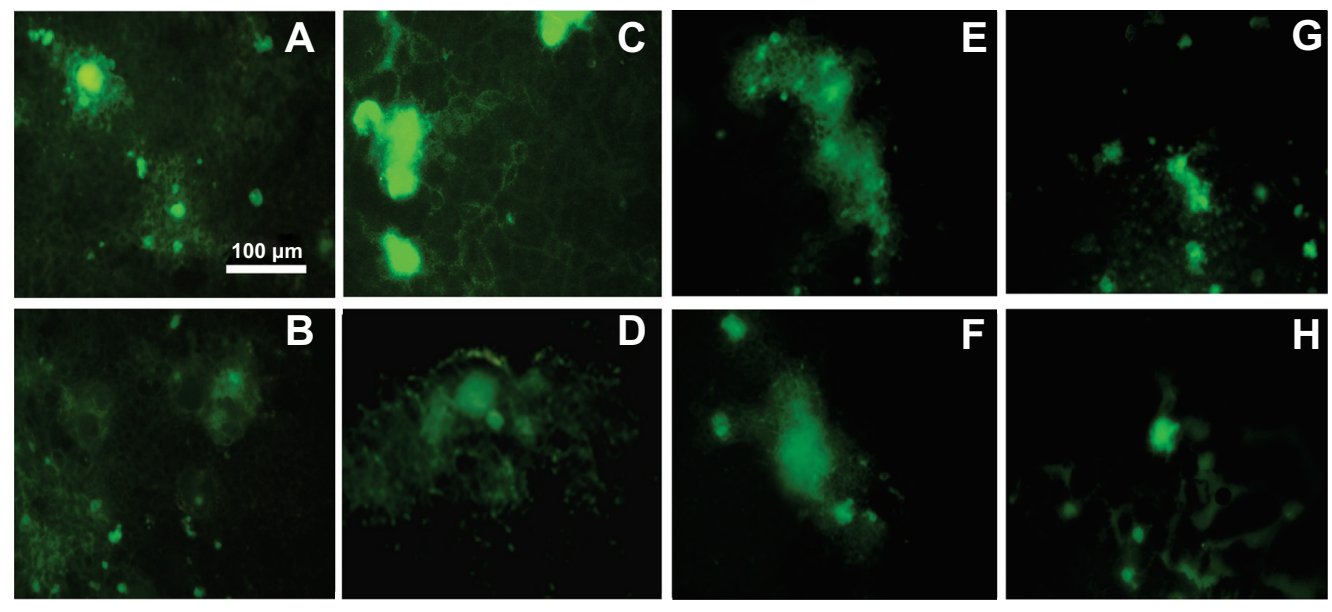

Figure S2 Cellular accumulation of FITC-labeled nanomicelles over 24 hours of incubation under various conditions: $(\mathbf{A})$ at $4^{\circ} \mathrm{C},(\mathbf{B})$ at $37^{\circ} \mathrm{C},(\mathbf{C})$ without cyclosporine $\mathrm{A}$, (D) with cyclosporine $\mathrm{A},(\mathbf{E})$ without $0.45 \mathrm{M}$ sucrose, $(\mathbf{F})$ with $0.45 \mathrm{M}$ sucrose, $(\mathbf{G})$ without brefeldine $\mathrm{A},(\mathbf{H})$ with brefeldine $\mathrm{A}$.

Notes: Representative images from three independent studies are shown. 

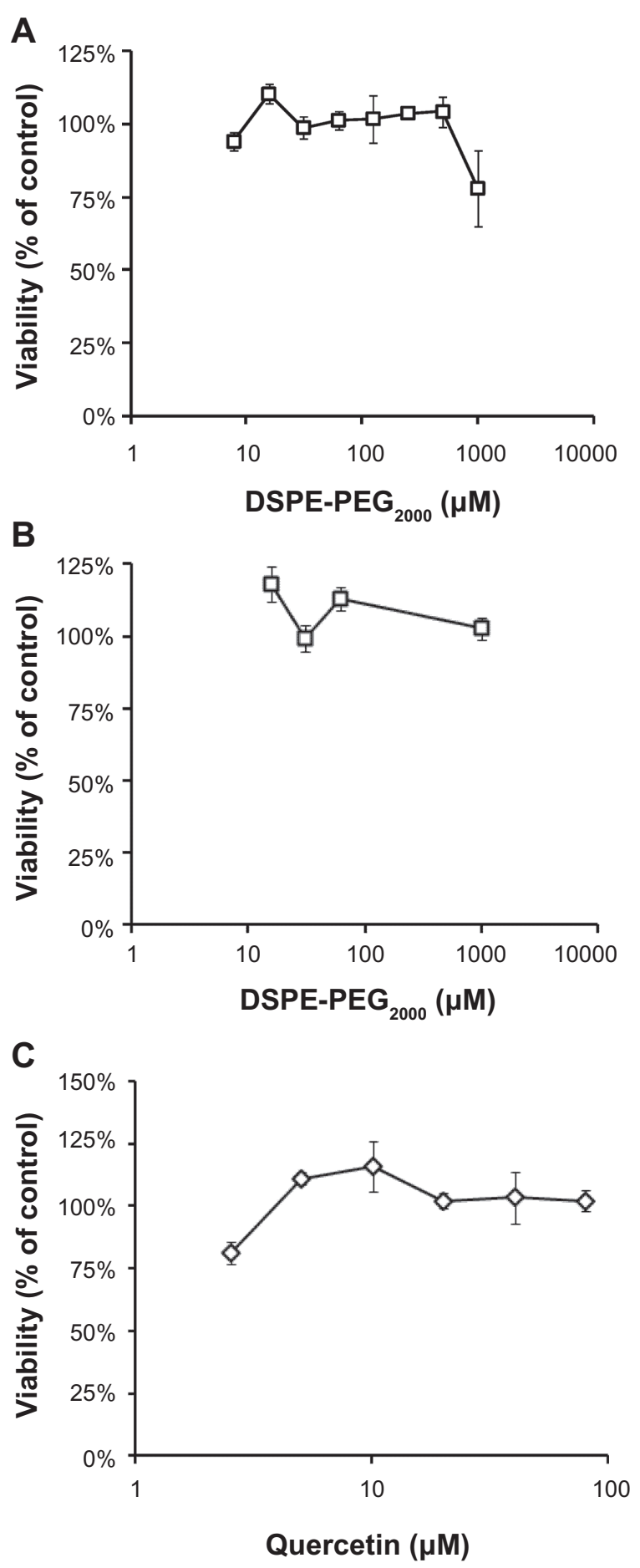

Figure S3 MTT viability of (A) empty, non-drug loaded nanomicelles, (B) quercetin-loaded nanomicelles (showing micelle concentrations), and (C) free quercetin in Caco-2 cell monolayer upon $24 \mathrm{~h}$ of exposure.

International Journal of Nanomedicine

Dovepress

\section{Publish your work in this journal}

The International Journal of Nanomedicine is an international, peerreviewed journal focusing on the application of nanotechnology in diagnostics, therapeutics, and drug delivery systems throughout the biomedical field. This journal is indexed on PubMed Central, MedLine, CAS, SciSearch ${ }^{\circledR}$, Current Contents ${ }^{\circledR} /$ Clinical Medicine,
Journal Citation Reports/Science Edition, EMBase, Scopus and the Elsevier Bibliographic databases. The manuscript management system is completely online and includes a very quick and fair peer-review system, which is all easy to use. Visit http://www.dovepress.com/ testimonials.php to read real quotes from published authors.

\footnotetext{
Submit your manuscript here: http://www.dovepress.com/international-journal-of-nanomedicine-journal
} 